\title{
The Coexistence of Hosts with Different Abilities to Discriminate against Cheater Partners: An Evolutionary Game-Theory Approach
}

\author{
Brian S. Steidinger ${ }^{\star}$ and James D. Bever \\ Department of Ecology, Evolution, and Behavior, Indiana University, Bloomington, Indiana 47405 \\ Submitted April 9, 2013; Accepted December 27, 2013; Electronically published April 11, 2014
}

\begin{abstract}
Evolutionary theory predicts that mutualisms based on the reciprocal exchange of costly services should be susceptible to exploitation by cheaters. Consistent with theory, both cheating and discrimination against cheaters are ubiquitous features of mutualisms. Several recent studies have confirmed that host species differ in the extent that they are able to discriminate against cheaters, suggesting that cheating may be stabilized by the existence of susceptible hosts (dubbed "givers"). We use an evolutionary gametheoretical approach to demonstrate how discriminating and giver hosts associating with mutualist and cheater partners can coexist. Discriminators drive the proportion of cheaters below a critical threshold, at which point there is no benefit to investing resources into discrimination. This promotes givers, who benefit from mutualists but allow cheater populations to rebound. We then apply this model to the plant-mycorrhizal mutualism and demonstrate it is one mechanism for generating host-specific responses to mycorrhizal fungal species necessary to generate negative plant-soil feedbacks. Our model makes several falsifiable, qualitative predictions for plant-mycorrhizal population dynamics across gradients of soil phosphorus availability and interhost differences in ability to discriminate. Finally, we suggest applications and limitations of the model with regard to coexistence in specific biological systems.
\end{abstract}

Keywords: host sanctions, mutualism, mycorrhiza, plant-soil feedbacks, species coexistence, soil phosphorus.

\section{Introduction}

Every reciprocal partnership is susceptible to exploitation (Yu 2001). This is particularly true in horizontal mutualisms where partners are able to disperse among neighboring hosts, a phenomenon that decouples host and partner fitness. Evolutionary theory predicts that horizontal mutualisms should be unstable due to fitness incentives for partners to defect or exploit the benefits of mutualism without investing resources. Although there are special sit-

\footnotetext{
* Corresponding author; e-mail: bsteidin@indiana.edu.
}

Am. Nat. 2014. Vol. 183, pp. 762-770. (C) 2014 by The University of Chicago. 0003-0147/2014/18306-54600\$15.00. All rights reserved.

DOI: $10.1086 / 675859$ uations where horizontal mutualisms are stabilized against cheaters, such as when partners are close kin (Hamilton 1964) or when repeat encounters with the same partners provide incentives to cooperate (Axelrod and Hamilton 1981), many diverse and ubiquitous mutualisms lack these qualities (Douglas 2008; Kiers and Dennison 2008). Nevertheless, horizontal mutualisms are both common in nature and persistent over evolutionary time, even in the presence of specialized cheaters. These mutualisms are stabilized by host discrimination, where a host invests resources into maintaining an environment that selectively promotes cooperative partners (Archetti et al. 2011).

In plants, mechanisms of discrimination include creating a chemical environment that is toxic to cheaters (Ruby and McFall-Nagi 1999), selectively aborting modular structures colonized by cheaters (Pellmyr and Huth 1994), and preferentially allocating resources to cooperative partners (Bever et al. 2009; Kiers et al. 2011). For simplicity, we call hosts that employ these mechanisms "discriminators," while hosts that forgo discrimination are called "givers." The existence of discrimination mechanisms across so many mutualisms presents a paradox-if discrimination is effective, why are there cheaters, and if there are no cheaters, why maintain traits for discrimination?

Foster and Kokko (2006) provide one answer to this paradox-host discrimination can be stabilized in populations where mutation/immigration reintroduces variability in the partner population's tendency to cooperate (or cheat). However, this seeded variability is only one mechanism for maintaining cheaters in the partner population. An alternative mechanism is one that involves negative feedbacks among coexisting species, such that giver hosts act as a susceptible reservoir for the production of cheater partners, while discriminator hosts sustain sufficient mutualists to prevent cheaters from driving the givers extinct. Indeed, the requisite cheaters and variability in host species' ability to discriminate is documented in 
the mutualisms between figs and pollinator wasps (Jandér and Herre 2010), Acacia and herbivore defense ants (Heil et al. 2009), and plants and mycorrhiza that enhance nutrient acquisition (Bever et al. 2009; Kiers et al. 2011; Grman 2012).

Fitness trade-offs between discriminating against cheaters and deriving benefit from cooperative partners may help level the playing field among host species. Several recent studies suggest that discriminating hosts derive relatively less benefit from cooperative partners than their giving competitors. For example, fig species associated with strong sanctions against cheater wasps have floral traits that reduce their maximum achievable seed yield relative to figs that tolerate cheating (Herre 1989; Jandér and Herre 2010). Acacia species that are better at promoting vigilant defensive ant species invest more resources into costly extrafloral nectaries than species that tolerate cheater, nondefensive ants (Heil et al. 2009). Plant hosts capable of reducing carbon allocation to mycorrhiza in conditions where the mycorrhiza do not promote growth and nutrition derive less benefit from mycorrhiza in conditions when the fungi cooperate (Grman 2012; Grman and Robinson 2013). In these examples, discrimination is a costly bet-hedging strategy that reduces both the harm of associating with a cheater partner and the benefit of associating with a cooperative partner.

The same discrimination that ensures hosts benefit from mutualist partners' function has the ability to increase the density of mutualists relative to cheaters in the partner population. This represents a noninclusive benefit of discrimination, as it can be exploited by hosts that benefit from cooperative partners without paying the cost of discrimination (the givers). However, while giver hosts reap greater rewards from cooperative partners, they are also defenseless against exploitation by cheaters. A combination of discrimination trade-offs and impacts on the partner community have the potential to promote negative density dependent feedback, where both discriminating and giving hosts alter the partner population in ways that benefit their competitors more than themselves.

Here we define an evolutionary game-theory model to show how the costs of discrimination can lead to the coexistence of discriminating and giving hosts and cooperative and cheating partners. We then apply our model to the plant-mycorrhizal mutualism and use it to develop qualitative predictions about population dynamics across gradients of soil resource availability. Finally, we suggest future applications of the model.

\section{General Model Framework}

Game-theoretical assumptions are that the population is infinite, phenotypes breed true, and fitness is determined through interactions with other phenotypes. We are using an asymmetrical model, where hosts and partners represent exclusive guilds. Hosts are considered to have little or no incentive to cheat, while partners have high incentives to cheat. This is the case for large hosts associating with multiple smaller partners with a higher intrinsic reproductive rate and ability to disperse propagules to neighboring hosts (Douglas 2008).

The players in our game are discriminator and giver hosts and mutualist and cheater partners. In order to keep track of host versus partner payoffs and proportions, we adopted a notation of capital letters for hosts and lowercase letters for partners. Giver hosts receive a benefit $(B)$ from associating with a mutualist and pay a cost $(K)$ when associating with a cheater. In contrast, discriminators experience a functional trade-off that reduces the benefit of associating with a mutualist partner and decreases the harm of associating with a cheater (fig. $1 A$ ). This is achieved mathematically by dividing the benefit and cost terms by the quotients $(1+r)$ and $(1+\beta r)$, respectively, where $r$ represents the magnitude of the trade-off (high $r=$ strong trade-off) and $\beta$ is a scalar term that determines the proportionality of the reductions in $K$ and $B$ (fig. $1 A$, $1 B$ ). For example, when $r=1$, a discriminating host receives half the maximum benefit $(B)$ from associating with a mutualist partner. When $\beta=1$, this results in a similar halving of the cost $(K)$ of associating with a cheater, while a $\beta<1$ results in a proportionally larger reduction in benefit relative to cost.

Partner strategies differ from hosts principally in having an incentive to cheat. Mutualist partners invest resources $(z)$ into and receive a benefit $(b)$ from their hosts. Cheater partners reap a benefit from giver hosts without paying a cost, but receive a benefit divided by the quotient $(1+$ $\alpha r$ ) when associating with a discriminator, where $\alpha$ is an additional scalar term that determines how strongly the host fitness trade-off influences cheater partner fitness (fig. $1 C, 1 D)$. Thus, $\alpha>0$ results in reductions in cheater fitness with increases in discrimination $(r)$. For simplicity, we will consider a case where increases in the host trade-off $r$ results in a reduction in the fitness of cheaters and a proportional decrease in the benefits and costs of associating with mutualists and cheaters ( or $\beta=\alpha=1$ ). These fitness payoffs can be represented in the form of a payoff matrix (table 1).

The fitness of each phenotype is equal to their per capita growth rates and is determined by taking the sum of their expected payoffs, weighted by the frequency of those payoffs. For instance, the fitness of the mutualist partner, $W_{\mathrm{m}}$, can be determined from the following equation:

$$
W_{\mathrm{m}}=D \times \mathrm{E}(m, D)+(1-D) \mathrm{E}(m, G),
$$

where $\mathrm{E}(m, D)$ and $\mathrm{E}(m, G)$ are the expected payoffs to 

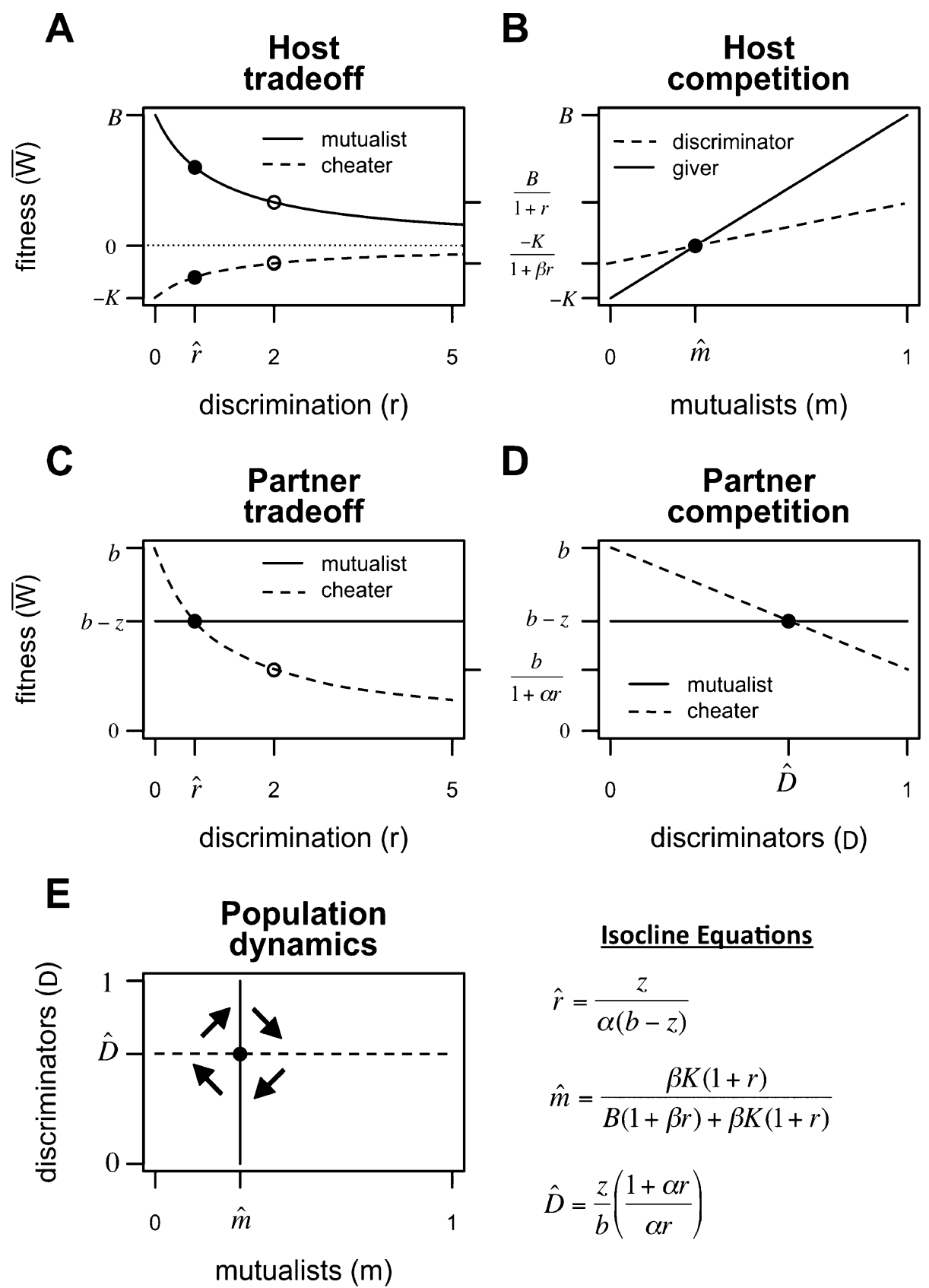

$$
\begin{aligned}
& \hat{r}=\frac{\underline{\text { Isocline Equations }}}{\alpha(b-z)} \\
& \hat{m}=\frac{\beta K(1+r)}{B(1+\beta r)+\beta K(1+r)} \\
& \hat{D}=\frac{z}{b}\left(\frac{1+\alpha r}{\alpha r}\right)
\end{aligned}
$$

mutualists $(\mathrm{m})$

Figure 1: $A$, Fitness benefit $(y)$ to the host of associating with a mutualist and cheater as a function of discrimination $(r)$, with the critical level of discrimination required to give mutualist partners a competitive advantage indicated by $\hat{r}$ and an arbitrary large value of $r=2$ indicated as an example. $B$, Fitness of discriminator (at $r=2)$ and giver hosts as a function of mutualist partners, with the intersection point marked $\hat{m}$. $C$, Fitness benefit to the partners of being a mutualist or cheater on a plant with discrimination $r$, with the critical level of discrimination indicated by $\hat{r}$ and the arbitrary level of $r=2$ indicated as in $A$. D. Fitness of mutualist and cheater partners as a function of discriminator hosts, with the intersection point marked $\hat{D}$. E, Cyclical population dynamics of partners ( $X$-axis) and hosts $(Y$-axis) indicated by vector lines when both $\hat{m}$ and $\hat{D}$ are between 0 and 1 . To the right of $E$, the equations for $\hat{r}, \hat{m}$, and $\hat{D}$ are indicated. $B, D$, and $E: b=B=5, z=K=2, r=2$, and $\alpha=\beta=1$. 
Table 1: Expected payoffs for hosts and partners

\begin{tabular}{llll}
\hline & Discriminator $(D)$ & \multicolumn{1}{c}{ Giver $(1-D)$} & \multicolumn{1}{c}{ Partner fitness } \\
\hline Mutualist $(m)$ & $b-z ; \frac{B}{1+r}$ & $b-z ; B$ & $W_{\mathrm{m}}=b-z$ \\
Cheater $(1-m)$ & $\frac{b}{1+\alpha r} ; \frac{-K}{1+\beta r}$ & $b ;-K$ & $W_{\mathrm{c}}=b\left[1-D\left(\frac{1}{1+\alpha r}\right)\right]$ \\
Host fitness & $W_{D}=\frac{m B}{1+r}-\frac{(1-m) K}{1+\beta r}$ & $W_{\mathrm{G}}=m(b+k)-k$ & \\
\hline
\end{tabular}

Note: Expected payoff bimatrix for partners (rows, left element) and hosts (columns, right element). Terms $B$ and $b$ are the benefit to the mutualism to hosts and partners, respectively, while $z$ is the cost of the mutualism to the partner and $K$ is the cost to the host of associating with a cheater. These terms are expressed in units of per capita growth rate, while the extent of host discrimination $(r)$ and the terms $\alpha$ and $\beta$ are scalars that adjust the fitness of discriminator hosts and/or cheater partners.

the mutualist partner when associating with discriminator $(D)$ and giver $(G=1-D)$ hosts, respectively. In this case, the fitness equation simplifies to $W_{\mathrm{m}}=b-z$. The fitness equations of each phenotype are calculated in the same manner (table 1). Note that the fitness of each strategy $x$ is is denoted as $W_{x}$.

The increase in the proportion of discriminator hosts and mutualist partners is determined from the replicator equation (Hofbauer and Sigmund 1998), such that

$$
\begin{aligned}
& \frac{d m}{d t}=m(1-m)\left(W_{\mathrm{m}}-W_{\mathrm{c}}\right), \\
& \frac{d D}{d t}=D(1-D)\left(W_{D}-W_{G}\right),
\end{aligned}
$$

for mutualists and discriminators, respectively.

One potential outcome of this model is coexistence via stable oscillations around an equilibrium point, which will occur whenever the zero growth isoclines for both host and partner strategies intersect (i.e., fall between 0 and 1; fig. $1 E$ ). To determine the zero growth isocline for the hosts, we set the rate of change for host strategies equal to $0(d D / d t=0)$ and solve the equality of both host fitnesses $\left(W_{D}=W_{G}\right)$ in terms of the proportion of mutualists $(\hat{m}$; fig. $1 B)$. In this case,

$$
\hat{m}=\frac{\beta K(1+r)}{B(1+\beta r)+\beta K(1+r)} .
$$

The host isocline $(\hat{m})$ will fall between 0 and 1 whenever all of the model terms are positive, a situation consistent with partners capable of either benefiting $(B>0)$ or harming $(K>0)$ hosts and discriminators experiencing both reduced benefit $(r>0)$ and harm $(\beta>0)$ from mutualist and cheater partners, respectively. If we do the same thing for the zero growth isocline of the partners, we can solve for $W_{\mathrm{m}}=W_{\mathrm{c}}$ in terms of the proportion of discriminator hosts, or

$$
\hat{D}=\frac{z}{b}\left(\frac{1+\alpha r}{\alpha r}\right)
$$

Thus, the position of the partner isocline is dependent on the cost to benefit ratio of mutualism $(z / b)$ and the degree to which discriminating hosts reduce the benefit of cheating (proportional to $\alpha r$ ). Only discrimination beyond a critical level $(\hat{r})$ is sufficient to reduce the benefit of cheating below that of mutualism (fig. $2 C$ ), where

$$
\hat{r}=\frac{z}{\alpha(b-z)} .
$$

If we consider a case where discrimination exceeds $\hat{r}$, then the position of the partner zero growth isocline $(\hat{D})$ will fall between 0 and 1 (fig. $1 D$ ). In addition, $\hat{D}$ will fall between 0 and 1 only when the benefit of the mutualism to the partners is greater than its cost $(b>z)$. The existence of an interior equilibrium point results in a population dynamic where discriminating hosts $(r>\hat{r})$ have an advantage when mutualists are below the host isocline $(m<\hat{m})$, while less discriminating hosts $(r<\hat{r})$ have an advantage when mutualists are above the host isocline $(m>\hat{m})$. The interior equilibrium point exhibits neutral stability, such that the populations will cycle around it without convergence or divergence (fig. 1E). This oscillating population dynamic is analogs to that which occurs in the familiar "battle of the sexes" model (Dawkins 1976), where "coy" (discriminating) females demand a costly courtship before copulation to insure they are matched with "faithful" (mutualist) males, while "quick" (giver) females do not demand courtship but are susceptible to "philanderer" (cheater) males (see Schuster and Sigmund 1981).

In contrast to the interior equilibrium that results in oscillating host and partner populations, there is an alternative monomorphic (or Nash) equilibrium. Specifically, when the benefit of mutualism is less than or equal to the cost $(b \leq z)$, only cheating is a viable strategy. If 

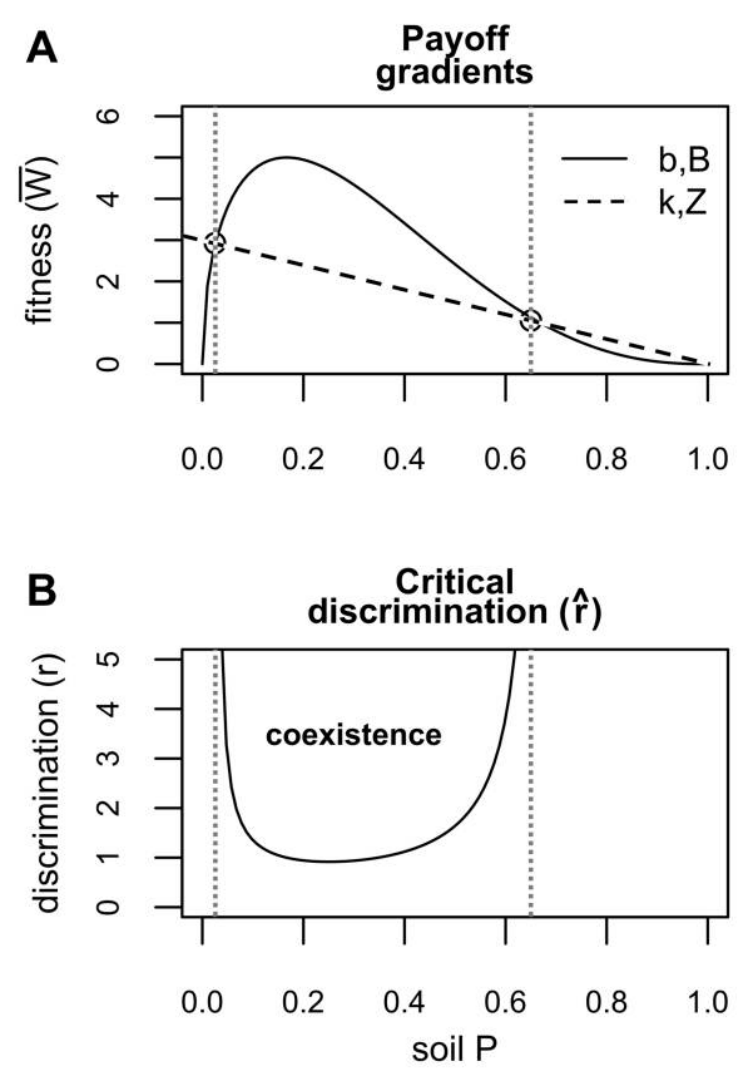

Figure 2: $A$, Terms in the payoff matrix shown graphically as a function of soil $\mathrm{P}$, with the benefit to the plant hosts and mycorrhizal fungi partners ( $B$ and $b$ ) expressed as a right tailed unimodal curve and the host cost of associating with a cheater $(K)$ and the cost of being a mutualist mycorrhizal fungi $(z)$ indicated by a straight line with negative slope, and the intersections indicated with vertical dotted lines. The values are $b_{\max }=B_{\max }=5$ and $k_{\max }=Z_{\max }=3$. $B$, Critical level of discrimination $(\hat{r})$ as expressed as a function of soil $\mathrm{P}$, with the asymptotes where $b=z$ indicated with vertical dotted lines. Population dynamics leading to coexistence are possible when discriminators have $r$ values above the black line.

the cost of associating with a cheater is greater than 0 $(K>0)$, then an unbeatable host strategy would be a discriminator that minimizes this cost $(r=\infty)$. Thus, in our model, all cases where coexistence is impossible lead to the breakdown of mutualism, with fixation of cheating partners and unresponsive hosts.

\section{Applying the Model to the Mycorrhizal Mutualism}

In order to illustrate the utility of the model, we will apply it to a specific mutualism. We use the association between approximately $80 \%$ of all vascular plants and arbuscular mycorrhizal (AM) fungi as a model system (Smith and Read 2008), as it is pervasive, fits model assumptions, and allows us to constrain variability in payoff matrix terms along gradients of resource availability.

Arbuscular mycorrhizal fungi live inside plant roots and feed entirely on carbon (C) provided by their host. In return, they produce filamentous networks of soil hyphae that acquire nutrients and transfer them to the host plant. Although the mutualism is multifunctional, the primary exchange of resources is the allocation of host $\mathrm{C}$ for soil phosphorus $(\mathrm{P})$, with as much as $80 \%$ of a host plant's total $\mathrm{P}$ acquired via its mycorrhizal partner (Marschner and Dell 1994).

In keeping with model assumptions, there is an asymmetry between host and partner incentives to cheat. Arbuscular mycorrhizal fungi have a large incentive to cheat (high cost of mutualism), with a steep trade-off between providing host benefits via nutrient acquisition and enhancing their own competitive ability within a host root (Bennett and Bever 2009; Bever et al. 2009). Consistent with this finding, several generalist mycorrhizal species have evolved to exploit plant $\mathrm{C}$ without providing any benefits (Graham and Abbott 2000; Burleigh et al. 2002; Smith et al. 2003, 2004; Koch et al. 2006; Violi et al. 2007). In contrast, although plants provide up to $20 \%$ of their fixed $\mathrm{C}$ to their mycorrhizal partners, the benefits of acquiring limiting soil $\mathrm{P}$ can result in a net increase in photosynthetic rate that more than defrays this cost (Kaschuk et al. 2009), such that plant cheaters appear to be restricted to highly specialized mycoheterophic species that specialize in low-light environments in the forest understory (Bidartondo 2005). In this context, allocating C to mycorrhizal fungi is costly only if the fungal partner fails to offset this investment with a sufficient augmentation of host photosynthetic rate; that is, when a plant associates with cheater fungi (with cost $K$ ).

In order to make our model tractable, we can constrain variability among the terms in the expected payoff matrix along a resource gradient. This has the benefit of providing predictions for both descriptive and experimental ecologists. For the plant mycorrhizal mutualism, we do this by defining the terms in the payoff matrix (table 1) as functions of soil $\mathrm{P}$ that approximate qualitative findings in the literature. Although these functions are somewhat crude approximations, we restrict their use to determining the relative fitness of host/partner strategies. The efficacy of this approach can be evaluated based on its ability to derive some of the known qualities of the plant-mycorrhizal fungi mutualism within the model framework.

First we define soil $\mathrm{P}$ as a level between 0 and 1, with 0 representing the most impoverished soils on earth (e.g., southern Australia) and 1 the most P-enriched soils (agricultural and young, newly exposed soils). Plant host benefits $(B)$ have been demonstrated to peak on soils with low level of $\mathrm{P}$ and decrease as soil $\mathrm{P}$ increases (Mosse 1973; 
Stribley et al. 1980; Johnson 1993; Treseder 2004; Hoeksema 2010). However, both experimental and agricultural studies demonstrate that mycorrhiza fail to provide any benefit to their hosts at extremely low levels of $\mathrm{P}$ availability (Koide and Li 1991; Ryan and Angus 2003). Thus, the function for plant benefit, $B(\mathrm{P})$, can be represented by a right-tailed unimodal curve, with high benefits on low$\mathrm{P}$ soils dropping off gradually as soil $\mathrm{P}$ increases and more abruptly on extremely P deficient soils. Mycorrhizal fungal benefit is assumed to be proportional to plant benefit (see Olsson et al. 2010) and is defined by the same function $(b(\mathrm{p})=B(\mathrm{p}))$ (fig. $2 A$; appendix).

We hypothesize that both the cost to the plant of being exploited by a cheater mycorrhiza $(K)$ and the cost of cooperation to mutualist mycorrhiza $(z)$ decrease linearly as soil $\mathrm{P}$ increases. This is consistent with the qualitative findings that plant hosts can meet $\mathrm{P}$ demands on highly fertile soils without fungal assistance and mutualist fungi pay a higher cost to deliver $\mathrm{P}$ to plants when it is less concentrated in the soil (reviewed by Johnson et al. 1996). Thus, $z(\mathrm{P})=K(\mathrm{P})=(1-\mathrm{P}) k_{\max }$, with $k_{\max }$ equal to both the maximum costs of being exploited by a cheater mycorrhiza and of providing growth and nutritional benefits to a plant host.

Consistent with the general model predictions, coexistence is only possible when the critical level of discrimination is finite, which only occurs when the benefit of the mutualism exceeds its cost $(b>z)$ to the mycorrhizal fungi $(2 B)$. This occurs at intermediate levels of the soil P gradient, with the critical level of discrimination $(\hat{r})$ reaching infinity at very low and high levels of soil P. Infinite discrimination, in turn, signals the breakdown of the symbioses, as hosts can limit fitness losses only to cheater partners. From a plant physiological point of view, this is consistent with the benefits of nonmycotrophy at the extreme ends of the soil $\mathrm{P}$ gradient.

\section{Discussion}

This model illustrates that hosts with different capacities to discriminate against cheaters can coexist via negative feedback on their partner communities. This is due to the non-inclusive benefits of altering the composition of a partner community, where givers benefit from but ultimately reverse discriminator's costly augmentation of mutualist partners. While Foster and Kokko (2006) have demonstrated the importance of variability in partner cooperation to maintain host discrimination, our model proposes that cheaters can facilitate host species coexistence. The existence of host species with different capacities to discriminate against cheater partners has recently been demonstrated to occur in a wide variety of mutualisms (Pellmyr 1994; Keirs et al. 2007; Bever et al. 2009;
Heil et al. 2009; Jandér and Herre 2010; Kiers et al. 2011; Grman 2012), suggesting these population dynamics may be a general feature of mutualisms. In this context, giving and discriminating hosts act as reservoirs of the cheater and mutualist partners each requires to coexist.

The stable oscillations generated by our model should contribute to conspecific negative density dependence (CNDD), where species alter their local environments in ways that depress their population growth rates. The effects of CNDD are proposed to be a powerful mechanism of species coexistence, particularly in diverse tropical forests (Janzen 1970; Connell 1971; Mangan et al. 2010) and, more recently, broadly across North American forests (Packer and Clay 2000; Nakashizuka 2001; HilleRisLambers et al. 2002; McCarthy-Neumann and Kobe 2010; Johnson et al. 2012). However, while CNDD is thought to occur via the accumulation of species-specific pathogens and predators, our model generates these effects via hosts' impacts on partner communities that include cheaters. Thus, discriminators augment the population of mutualist partners until cheating is too rare to justify the cost of discrimination, which promotes the greater relative population growth of giver hosts. When giver hosts reach a critical density, it pays for partners to cheat, and the cheater population increases.

We provided an example of how to apply our model to the mutualism between plants and arbuscular mycorrhizal fungi. By constraining variability of our model terms along a resource gradient, we were able to provide several qualitative findings, as well as reproduce important features of the mutualisms.

\section{Examples of Model Application}

In the plant-mycorrhizal mutualism, we found that soils with low to intermediate levels of soil $\mathrm{P}$ should support the coexistence of plant hosts and mycorrhizal fungi with different tendencies to discriminate or cheat, respectively. Fungal cheating and plant discrimination should become winning strategies on both low- and high-P soils where the cost of mutualism exceeds its benefit $(z>b)$. These findings are consistent with the absence of mycorrhizal plants on the most impoverished soils on the globe (Lambers et al. 2008) as well as the tendency of very P-rich agricultural soils to support cheater strains of mycorrhiza (Verbruggen and Kiers 2010).

The population oscillations in our model are analogous to negative plant soil feedbacks, where fungi that accumulate around conspecific plant hosts depress plant performance relative to fungi from heterospecific plant hosts (Bever 1999, 2002a, 2002b; Casper and Castelli 2003). Our model demonstrates that trade-offs in the ability to promote mutualist partners and the benefit derived from those 
partners are one potential mechanism to generate mycorrhiza-mediated negative plant soil feedbacks. Further, a closer examination of at least one frequently cited example of negative plant soil feedback provides some support for our model. Bever (2002a) found evidence for negative feedbacks between Plantago, a highly mycotrophic forb, and Panicum, a significantly less responsive grass, by gauging plant performance with either conspecific or heterospecific live soil inocula. Further, they found evidence that the feedbacks were caused by the accumulation for different arbuscular mycorrhizal fungal species in the rhizosphere of the plant hosts.

Several of the findings from the Bever et al. (2002a) study are qualitatively consistent with our model, including evidence that (i) discriminators plants experience a trade-off in mycorrhizal responsiveness that negatively impacts the fitness of cheater fungi, (ii) cheaters depress the growth of highly responsive givers relative to less responsive discriminators, and (iii) discriminators alter their partner communities in ways that benefit givers more than themselves. Thus, (i) the least beneficial mycorrhizal fungal species accumulated around the highly mycotrophic Plantago (giver) but not the less responsive Panicum (discriminator); (ii) this fungal strain depressed the growth of Plantago (giver) but not Panicum (discriminator); and (iii) in contrast, two mycorrhizal fungal species that were highly beneficial to Plantago (giver) but had only slightly positive and/or negative to Panicum (discriminator) were accumulated in the soil of Panicum. This example suggests that the trade-offs required to generate coexistence in our model are realized among competing host species.

Another potential application of our model is with respect to the coexistence of highly mycotrophic warm $\left(\mathrm{C}_{4}\right)$ and lesser or nonmycotrophic cool $\left(\mathrm{C}_{3}\right)$ season grasses in tall grass prairie ecosystems. Recent work has demonstrated that when fertilization reduces the benefit of mycotrophy, $\mathrm{C}_{3}$ plants have reduced levels of mycorrhizal colonization, while $\mathrm{C}_{4}$ grasses are nonresponsive (Grman 2012; Grman and Robinson 2013). Thus, one could hypothesize that $\mathrm{C}_{3}$ grasses are candidate discriminators, while $\mathrm{C}_{4}$ grasses are candidate givers. However, as yet there is no evidence that the reduction in mycorrhizal colonization in $\mathrm{C}_{3}$ grasses reduces the fitness of cheater more strongly than mutualist mycorrhizal fungi. With respect to our model, this would involve determining the sign and magnitude of the scalar term $\alpha$, which must be positive and sufficiently large to reduce cheater fungi's competitive advantage relative to mutualists. An alternative strategy would be for plants to minimize the harm of cheater fungi without punishing them in the form of precise sanctions (e.g., $\beta>0$ decreases the harm of cheating; $\alpha \leq 0$ fails to punish cheaters).

\section{Limitations and Future Directions}

Although we evaluate only one trade-off axis in our model (responding to mutualists vs. reducing the harm of cheaters), it is likely that an additional trade-off exists between host responsiveness to mutualist partners $(B)$ and the ability of the host to acquire resources via a nonsymbiotic pathway. Thus, while $\mathrm{C}_{3}$ grasses are less responsive to mycorrhizal fungi than $\mathrm{C}_{4}$ grasses, they also tend to develop finer and more branched root systems that could increase their ability to acquire soil nutrients in the absence of fungal partners (Grman 2012). Similarly, plants growing on the most P-impoverished soils in the world develop specialized cluster-roots that are generally nonmycotrophic and adapted to acquire nutrients in recalcitrant chemical forms (Lambers 2008). This additional trade-off axis could complicate our model by requiring additional environmentally sensitive terms or altering the term $\alpha$ in unpredictable ways.

The plant mycorrhizal system is easily amenable to a model that involves feedbacks on hosts interacting with a shared partner community. This is due to the lack of specificity between mycorrhiza and plant hosts, which allows for host/partner feedbacks to act in a reciprocal fashion. Out model could work equally well for other mutualisms with low specificity of association, such as those between legumes and nitrogen-fixing rhizobium. In contrast, our model would have to altered to address the role of hostspecificity before it can be applied to highly coevolved plant-pollinator systems (e.g., those among fig/fig-wasps and yucca/yucca-moths). In these cases, the model in its current form can only describe feedbacks within species.

Our model integrates the problem of stability in mutualisms with the problem of diversity among coexisting hosts. It provides one potential explanation for the coexistence of host species that differ in their ability to discriminate against cheaters. While this range of discrimination strengths is a relatively recently documented phenomenon, we predict that, as species are known to differ in almost every observable respect, interspecies discrimination discrepancies will be revealed as a feature common to all mutualisms. The efficacy of the model will rest on experimental and descriptive studies of mutualisms where the natural history is well enough understood that these interspecies differences in discrimination can be controlled.

\section{Acknowledgments}

We would like to acknowledge the helpful advice of $M$. Kelly, A. Strauss, and P. Zee. The work was funded by a 
National Science Foundation graduate research fellowship, DEB-0919434, DEB-1050237, and ROI-GM092660.

\section{APPENDIX}

\section{Equations for Resource-Dependent Payoffs}

An equation describing a right-tailed, unimodal curve is used to specify the benefits of the plant hosts $(B)$ and fungal partners $(b)$ along a gradient of soil phosphorus (P) (fig. 2A). The functions are defined with respect to two parameters-their shape and maximum value. These two parameters can be measured empirically and influence both the position of the equilibrium points in our model and the range of soil $\mathrm{P}$ values that correspond to different population dynamics (coexistence vs. dominance of nonmycorrhizal plant hosts and cheater fungi).

First, we specify the shape of the relationship as

$$
b(\mathrm{P})=\mathrm{P}^{a}(1-\mathrm{P})^{b},
$$

where $a$ and $b$ determine the position and steepness of the peak. Next, we specify the maximum value for this function. This is done by normalizing the that the function $b(\mathrm{P})$ so that it has a range between 0 and 1 . Finally, we multiply the normalized function by our specified maximum value $b_{\max }$ so that the new function ranges between 0 and $b_{\max }$.

First, we determine the level of soil phosphorus where $b(\mathrm{P})$ peaks, which is found by solving for $\mathrm{P}$ when the derivative of $b(\mathrm{P})$ is set equal to 0 , or $\mathrm{P}=a /(a+b)$. Next, the function $b(\mathrm{P})$ is divided by $b[a c /(a+b)]$, which sets the maximum value equal to 1 . If we multiply the proportion of $b(\mathrm{P}) / b[a c /(a+b)]$ by our specified maximum value, we get

$$
\frac{b(\mathrm{P})}{b[a /(a+b)]} \times b_{\max }=\left(\frac{a}{a+b}\right)^{-a}\left(\frac{b}{a+b}\right)^{-b} \mathrm{P}^{a}(1-\mathrm{P})^{b} b_{\max }
$$

which is a distribution with a fully specified shape and maximum value. In figure $2 A$, the shape and maximum values of functions $b(\mathrm{P})$ and $B(\mathrm{P})$ are defined by $a=0.5$, $b=2$, and $b_{\max }=5$.

\section{Literature Cited}

Archetti M., S. Istvan, H. Moshe, M. E. Frederickson, N. E. Pierce, and D. W. Yu. 2011. Economic game theory for mutualism and cooperation. Ecology Letters 14:1300-1312.

Axelrod, R., and W. D. Hamilton. 1981. The evolution of cooperation. Science 211:1390-1396.

Bennett, A. E., and J. D. Bever. 2009. Trade-offs between arbuscular mycorrhizal fungal competitive ability and host growth promotion in Plantago lanceolata. Oecologia (Berlin) 160:807-816.

Bever, J. D. 1999. Dynamics within mutualism and the maintenance of diversity: inference from a model of interguild frequency dependence. Ecology Letters 2:52-62.

- 2002a. Host-specificity of AM fungal population growth rates can generate feedback on plant growth. Plant and Soil 244: 281-290.

-2002b. Negative feedback within a mutualism: host-specific growth of mycorrhizal fungi reduces plant benefit. Proceedings of the Royal Society B: Biological Sciences 269:2595-2601.

Bever, J. D., S. C. Richardson, B. M. Lawrence, J. Holmes, and M. Watson. 2009. Preferential allocations to beneficial symbiont with spatial structure maintains mycorrhizal mutualism. Ecology Letters 12:13-21.

Bidartondo, M. I. 2005. The evolutionary ecology of myco-heterotrophy. New Phytologist 167:335-352.

Burleigh, S. H. , T. Cavagnaro, and I. Jakobsen. 2002. Functional diversity of arbuscular mycorrhizas extends to the expression of plant genes involves in P nutrition. Journal of Experimental Botany 53:1593-1601.

Casteli, J. P., and B. B. Casper. 2003. Intraspecific AM fungal variation contributes to plant-fungal feedback in a serpentine grassland. Ecology 84:323-336.

Connell, J. H. 1971. On the role of natural enemies in preventing competitive exclusion in some marine animals and in rain forest trees. Pages 298-312 in P. J. Den Boer and G. Gradwell, eds. Dynamics of populations. Centre for Agricultural Publishing and Documentation, Wageningen.

Dawkins, R. 1976. The selfish gene. Oxford University Press, Oxford. Douglas, A. E. 2008. Conflict, cheats and the persistence of symbioses. New Phytologist 177:849-858.

Foster, K. R., and H. Kokko. 2006. Cheating can stabilize cooperation in mutualisms. Proceedings of the Roval Society B: Biological Sciences 273:2233-2239.

Graham, J. H., and L. K. Abbott. 2000. Wheat responses to aggressive and non-aggressive arbuscular mycorrhizal fungi. Plant and Soil 220:207-218.

Grman, E. 2012. Plant species differ in their ability to reduce allocation to non-beneficial arbuscular mycorrhizal fungi. Ecology 93: 711-718.

Grman, E., and T. M. P. Robinson. 2013. Resource availability and imbalance affect plant-mycorrhizal interactions: a field test of three hypotheses. Ecology 94:62-71.

Hamilton, W. D. 1964. The genetical evolution of social behavior. Journal of Theoretical Biology 239:195-202.

Heil, M., M. Gonzalez-Teuber, L. Clement, S. Kautz, M. Verhaagh, and J. Silva-Bueno. 2009. Divergent investment strategies of Acacia myrmecophytes and the coexistence of mutualists and exploiters. Proceedings of the National Academv of Sciences of the USA 106: 18091-18096.

Herre, E. A. 1989. Coevolution of reproductive characteristics in 12 species of new world figs and their pollinator wasps. Experentia 45:637-647.

HilleRisLambers, J., J. S. Clark, and B. Beckage. 2002. Densitydependent mortality and the latitudinal gradient in species diversity. Nature 417:732-735.

Hoeksema, J. D. 2010. A meta-analysis of context-dependency in plant response to inoculation with mycorrhizal fungi. Ecology Letters 13:394-407. 
Hofbauer, J., and K. Sigmund. 1998. Evolutionary games and population dynamics. Cambridge University Press, Cambridge.

Jandér, K. C., and E. A. Herre. 2010. Host sanctions and pollinator cheating in the fig tree-fig wasp mutualism. Proceedings of the Roval Society B: Biological Sciences 277:1481-1488.

Janzen, D. H. 1970. Herbivores and number of tree species in tropical forests. American Naturalist 104:501-528.

Johnson, D. J., W. T. Beaulieu, J. D. Bever, and K. Clay. 2012. Negative density dependence and forest diversity. Science 336:904-907.

Johnson, N. C. 1993. Can fertilization of soil select less mutualistic mycorrhizae? Ecological Applications 3:749-757.

Johnson, N. C., J. H. Graham, and F. A. Smith. 1996. Functioning of mycorrhizal associations along the mutualism-parasitism continuum. New Phytologist 135:575-585.

Kaschuk, G., T. W. Kuyper, P. A. Leffelaar, M. Hungria, and K. E. Giller. 2009. Are the rates of photosynthesis stimulated by the carbon sink strength of rhizobial and arbuscular mycorrhizal symbioses? Soil Biology and Biochemistry 41:1233-1244.

Kiers, E. T., and R. F. Dennison. 2008. Sanctions, cooperation, and the stability of plant-rhizosphere mutualisms. Annual Review of Ecology, Evolution, and Systematics 39:215-236.

Kiers, E. T., M. Duhamel, Y. Beesetty, J. A. Mensah, O. Franken, E. Verbruggen, C. R. Fellbaum, et al. 2011. Reciprocal rewards stabilize cooperation in the mycorrhizal symbiosis. Science 333:880882.

Kiers, E. T., M. G. Hutton, and R. F. Denison. 2007. Human selection and the relaxation of legume defenses against ineffective rhizobia. Proceedings of the Roval Societv B: Biological Sciences 274:31193126.

Koch, A. M., D. Croll, and I. R. Sanders. 2006. Genetic variability in a population of arbuscular mycorrhizal fungi causes variation in plant growth. Ecology Letters 9:103-110.

Koide, R. T., and M. Li. 1991. On host regulation of the vesiculararbuscular mycorrhizal symbiosis. New Phytologist 114:59-74.

Lambers, H., J. A. Raven, G. R. Shaver, and S. E. Smith. 2008. Nand $\mathrm{P}$-acquisition strategies change with soil age. Trends in Ecology and Evolution 23:95-103.

Mangan, S. A., S. A. Schnitzer, E. A. Herre, K. M. L. Mack, M. C. Valencia, E. I. Sanchez, and J. D. Bever. 2010. Negative plant-soil feedback predicts tree-species relative abundance in a tropical forest. Nature 466:752-755.

Marschner, H., and B. Dell. 1994. Nutrient uptake in mycorrhizal symbiosis. Plant and Soil 159:89-102.

McCarthy-Neumann, S., and R. K. Kobe. 2010. Conspecific plantsoil feedbacks reduce survivorship and growth of tropical tree seedlings. Lournal of Ecology 98:396-407.

Mosse, B. 1973. Plant growth responses to vescular-arbuscular my- corrhizas. IV. In soil given additional phosphorus. New Phytologist 72:127-136.

Nakashizuka, T. 2001. Species coexistence in temperate, mixed deciduous forests. Trends in Ecology and Evolution 16:205-210.

Olsson, P. A., J. Rahm, and N. Aliasgharzad. 2010. Carbon dynamics in mycorrhizal symbioses is linked to carbon costs and phosphorus benefits. FEMS Microbial Ecology 72:125-131.

Packer, A., and K. Clay. 2000. Soil pathogens and spatial patterns of seedling mortality in a temperate tree. Nature 404:278-281.

Pellmyr, O., and C. J. Huth. 1994. Evolutionary stability of mutualism between yuccas and yucca moths. Nature 372:257-260.

Ruby, E. G., and M. J. McFall-Nagi. 1999. Oxygen-utilizing reactions and symbiotic colonization of the squid light organ by Vibrio fischeri. Trends in Microbiology 7:414-420.

Ryan, M. H., and J. F. Angus. 2003. Arbuscular mycorrhizae in wheat and field pea crops on a low P soil: increase Zn-uptake but no increase in P-uptake or yield. Plant and Soil 250:225-239.

Schuster, P., and K. Sigmund. 1981. Coyness, philandering, and stable strategies. Animal Behavior 29:186-192.

Smith, S. E., and D. J. Read. 2008. Mycorrhizal symbiosis. 2nd ed. Academic Press, London.

Smith, S. E., F. A. Smith, and I. Jakobsen. 2003. Mycorrhizal fungi can dominate phosphate supply to plants irrespective of growth responses. Plant Physiology 133:16-20.

- 2004. Functional diversity in arbuscular mycorrhizal (AM) symbioses: the contribution of the mycorrhizal $\mathrm{P}$ uptake pathway is not correlated with mycorrhizal responses in growth or total $\mathrm{P}$ uptake. New Phvtologist 162:511-524.

Stribley, D. P., P. B. Tinker, and J. H. Rayner. 1980. Relation of internal phosphorus concentration and plant weight in plants infected by vesicular-arbuscular mycorrhizas. New Phytologist 86:261-266.

Treseder, K. K. 2004. A meta-analysis of mycorrhizal responses to nitrogen, phosphorus, and atmospheric $\mathrm{CO}_{2}$ in field studies. $\mathrm{New}$ Phvtologist 164:347-355.

Verbruggen, E., and E. T. Kiers. 2010. Evolutionary ecology of mycorrhizal functional diversity in agricultural systems. Evolutionary Applications 3:547-560.

Violi, H. A., K. K. Treseder, J. A. Menge, S. F. Wright, and C. J. Lovatt. 2007. Density dependence and interspecific interactions between arbuscular mycorrhizal fungi mediated plant growth, glomalin productions, and sporulation. Canadian Journal of Botany 85:63-75.

Yu, D. W. 2001. Parasites of mutualisms. Biological Journal of the Linnean Society 72:529-549.

Associate Editor: Vlastimil Křivan Editor: Susan Kalisz 